\title{
Hemoglobin Levels and Postoperative Outcome in Pediatric Surgical Patients
}

\author{
Claudine Kumba* \\ Department of Pediatric and Obstetric Anesthesia and Critical Care, Hôpital Universitaire Necker, France. \\ ORCID ID: https://orcid.org/0000-0002-9748-5141
}

\begin{abstract}
Background: Postoperative outcome in children is multifactorial. Among the reported predictors of postoperative outcome, preoperative anemia has been related to adverse outcome in children. A secondary analysis was undertaken to determine the correlation between hemoglobin levels and postoperative outcome in children included in a cohort of an observational pediatric study published previously since this analysis has not been done.
\end{abstract}

Objective: To determine the correlation between preoperative, intra-operative, postoperative hemoglobin levels and postoperative outcome in children in neurosurgery, abdominal and orthopedic surgery.

Methods: Secondary analysis of a sub-cohort of 252 pediatric surgical patients with a median age of 62 months [12.50-144.00].

Results: Preoperative hemoglobin levels were negatively correlated to length of stay in the intensive care unit (LOSICU) ( $\mathrm{p}=0.002)$, to length of hospital stay (LOS) $(\mathrm{p}<0.0001)$, to the number of patients with intra-operative and/or postoperative complications ( $\mathrm{p}<0.0001)$ and to re-surgery $(\mathrm{p}<0001)$. Low preoperative hemoglobin levels below $6 \mathrm{~g} / \mathrm{dL}$ were correlated to higher postoperative LOSICU and LOS. Intra-operative hemoglobin levels were negatively correlated to LOS $(\mathrm{p}<0.0001)$ and to the number of patients with intra-operative and/or postoperative complications $(\mathrm{p}=0.004)$. Low intra-operative hemoglobin levels below $5 \mathrm{~g} / \mathrm{dL}$ were correlated to higher LOS. Postoperative hemoglobin levels were positively correlated to LMV ( $\mathrm{p}=0.002)$.

Conclusion: Hemoglobin levels are among other multifactorial predictors of postoperative outcome in pediatric surgical patients emphasizing the importance of a global patient blood management implementation program to improve outcome in surgical children.

Keywords: Anemia, Hemoglobin levels, Pediatric surgery, Postoperative outcome, Patient blood management, Transfusion

\section{Introduction}

It has been evidenced that in pediatric neurosurgery, abdominal and orthopedic surgery, ASA score (American Society of Anesthesiologists score), transfusion, emergency surgery, age, type of surgery were independent predictive factors of postoperative outcomes in terms of organ dysfunction, length of intensive care unit stay (LOSICU), length of mechanical ventilation (LMV), length of hospital stay (LOS) and total length of hospital stay, (TLOS) (LO-
SICU+LOS) ${ }^{1-4}$ In a study where 594 surgical patients with a mean age of $90.86 \pm 71.80$ months were retrospectively included, transfusion concerned all type of blood products precisely packed red blood cells (PRBC), fresh frozen plasma (FFP) and concentrated platelets units (CPU). ${ }^{1}$ Immunomodulation and immune reactions have been evoked as explanations of transfusion related adverse outcome such as transfusion related lung injury (TRALI), transfusion associated cardio-circulatory overload (TACO). ${ }^{5}$ Goal directed transfusion protocols with point-of-care tests have demonstrated
Quick Response Code:

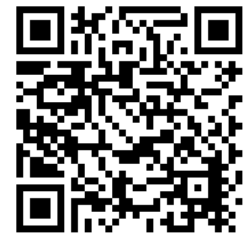

\footnotetext{
*Corresponding author: Claudine Kumba, Department of Pediatric and Obstetric Anesthesia and Critical Care, Hôpital Universitaire Necker Enfants Malades, Assistance Publique Hôpitaux de Paris, Université de Paris, 146 Rue de Sèvres, 75015 Paris, France

Received: 06 September, 2021

Published: 07 October, 2021

Citation: Kumba C. Hemoglobin Levels and Postoperative Outcome in Pediatric Surgical Patients. SOJ Pedia Clin Neonato. 2021;1(3):1-8. DOI: 10.53902/SOJPCN.2021.01.000511
} 
reduction of blood products administration such as fresh frozen plasma and reduction of LOS in pediatric hemorrhagic surgery. ${ }^{6}$ Anemia has been reported as a predictive factor of morbi-mortality in children., ${ }^{7,8}$ Weighing the benefits and risks of transfusion and anemia is mandatory to improve outcome in patients. A multicentre prospective study in pediatric intensive care patients demonstrated that a restrictive transfusion management in critically ill children did not increase adverse outcome compared to a liberal transfusion strategy. ${ }^{9}$ Transfusion triggers are not absolute and depend on patient status and clinical context. The aim of this article was to determine whether there was a correlation between preoperative, intra-operative and postoperative hemoglobin levels and postoperative outcome in terms of postoperative complications, re-surgery, mortality, LOSICU, LOS, TLOS and LMV in children included in the observational retrospective study since this data was not analyzed then. ${ }^{1}$

\section{Methods}

This is a secondary analysis of the database of 594 children included in the retrospective observational monocentric study from 1 January 2014 to 17 May 2017. ${ }^{1}$ This study was declared to the CNIL, National Commission for Computer Science and Liberties on 21 February 2017 under the registration number 2028257 v0 and received approval from the Ethics Committee of Necker on 21 March 2017 under the registration number 2017-CK-5-R1. ${ }^{1}$ The included patients were all children enrolled in the study and who had preoperative, intra- operative and postoperative hemoglobin levels available. The exclusion criteria were all patients enrolled in this study who did not have preoperative, intra-operative and postoperative hemoglobin levels. Statistical analysis was realized with XLSTAT 2020.4.1 software. Non normally distributed variables were compared with Mann-Whitney U test. Categorical variables were compared with Chi squared test or Fischer's exact test accordingly. Correlations between hemoglobin levels and outcomes were analyzed with linear regression. Non normally distributed variables were expressed in medians with interquartile ranges. Categorial variables were expressed in proportions. A p-value of less than 0.05 was considered significant.

\section{Results}

252 children with a median age of 62 months [12.50-144.00] were included from neurosurgery, abdominal and orthopedic surgery (Table 1). 195 children received PRBC and/or FFP and/or CPU intra-operatively and 57 children did not receive blood products. Median preoperative, intra-operative and postoperative hemoglobin levels were 11.65g/dL [10.38-12.90], 9g/dL [8-11.03], and $11.2 \mathrm{~g} / \mathrm{dL}$ [9.9-12.5] respectively (Table 2). Median preoperative hemoglobin levels were significantly higher than intra-operative $(p<0.0001)$ and postoperative hemoglobin levels $(p=0.013)$. Median LOSICU was 4 days [3-10], median LOS was 4 days [2-14.25], median TLOS was 9 days [5-26] and median LMV was 0 days [02] (Table 2). There were 39 ASA I (15.48\%), 50 ASA II (19.84\%), 122 ASA III (48.41\%), 36 ASA IV (14.29\%) and 5 ASA V (1.98\%) patients (Table 2). 83 (32.94\%) patients presented intra-operative or postoperative complications. Among these some had more than 1 complication (Table 3). 21 patients had re-surgery (8.33\%) and there were 7 deaths (Table 2). Complications are illustrated in Table 4 . There were 112 complications, some patients had more than 1 complication. The majority of these complications, 103 (91.96\%) occurred in transfused patients and $9(8.04 \%)$ in non-transfused patients. The most common intra-operative complication was hemorrhagic shock in 16 (6.35\%) patients, followed by broncho-laryngospasm in $3(1.19 \%)$ patients, followed by difficult intubation in $1(0.39 \%)$ patient, cardiac arrest in $1(0.39 \%)$ patient and anaphylaxis in $1(0.39 \%)$ patient. The majority of intra-operative complications occurred in transfused patients (Table 4).

The most common postoperative organ failure was neurologic in 18 (7.14\%) patients, followed by cardio-circulatory in $13(5.16 \%)$ patients, followed by respiratory in $12(4.76 \%)$ patients, followed by renal in 4 (1.59\%) patients, multi-organ in $4(1.59 \%)$ patients, followed by hepatic in $2(0.79 \%)$ patients, hemorrhagic shock in $1(0.39 \%)$ patient and anaphylaxis in $1(0.39 \%)$ patient. The majority of postoperative organ failure occurred in transfused patients (Table 4). The most common postoperative infection was septicemia in $10(3.97 \%)$ patients, followed by pulmonary sepsis in $8(3.17 \%)$ patients, followed by abdominal sepsis in $7(2.78 \%)$ patients, followed by urinary sepsis in $6(2.38 \%)$ patients, followed by neuro-meningeal sepsis in $2(0.79 \%)$ patients and surgical wound sepsis in $2(0.79 \%)$ patients. The majority of postoperative sepsis occurred in transfused patients (Table 4).

Table 3 illustrates general characteristics in transfused and non-transfusion patients. Patients in the transfusion group were significantly younger than in the no transfusion group. LOSICU, LMV, LOS and TLOS were significantly higher in the transfusion group than in the no transfusion group. The number of patients with re-surgery and complications were significantly higher in the transfusion group than in the no transfusion group. The number of deaths was not different between the two groups. The number of ASA III and ASA IV patients was significantly higher in the transfusion group than in the no transfusion group. Median preoperative and intra-operative hemoglobin levels were significantly lower in transfused patients $11 \mathrm{~g} / \mathrm{dL}[10-12.5]$ and $8.5 \mathrm{~g} / \mathrm{dL}$ [7.7-10.6] than in non-transfused patients $13.2 \mathrm{~g} / \mathrm{dL}[12.1-14.10]$ and $10.9 \mathrm{~g} / \mathrm{dL}$ [9.812.0] respectively $(\mathrm{p}<0.0001)$ (Table 3$)$. Postoperative hemoglobin levels were not different between the transfused patients $11.2 \mathrm{~g} /$ $\mathrm{dL}$ [9.85-12.6] and non-transfused patients 11.3g/dL[10.2-12.1] $(\mathrm{p}=0.89)$ (table 3). After linear regression, preoperative hemoglobin levels were negatively correlated to LOSICU (with $4 \%$ variability, $\mathrm{p}=0.002$ ), LOS (with 9\% variability, $\mathrm{p}<0.0001$ ), TLOS (with $8 \%$ variability, $\mathrm{p}<0.0001$ ) to the number of patients with intra-oper- 
ative and/or postoperative complications (with 6\% variability, $\mathrm{p}<0.0001$ ), to re-surgery (with 7\% variability, $\mathrm{p}<0001$ ) (Figures 1 and 2). There was no correlation between preoperative hemoglo- bin levels with LMV ( $\mathrm{p}=0.34)$ and mortality ( $\mathrm{p}=0.074)$ (Figures 1 and 2). Low preoperative hemoglobin levels below $6 \mathrm{~g} / \mathrm{dL}$ were correlated with high LOSICU, LOS and TLOS.

Table 1: Surgical Characteristics.

\begin{tabular}{|c|c|}
\hline Surgical Characteristic & Number of patients \\
\hline Ano-rectal malformation & 3 \\
\hline Neuroblastoma & 11 \\
\hline Liver transplantation & 19 \\
\hline Pelvic tumor & 4 \\
\hline Splenectomy & 1 \\
\hline Intestinal resection & 4 \\
\hline Hepatic tumor resection & 4 \\
\hline Vascular bypass & 4 \\
\hline Renal transplantation & 6 \\
\hline Duodeno-gastroscopy & 1 \\
\hline Pulmonary lobectomy & 1 \\
\hline Nissen gastrostomy & 1 \\
\hline Conjoined twins separation & 3 \\
\hline Kasai & 2 \\
\hline Exploratory Laparotomy & 1 \\
\hline Omphalocele & 2 \\
\hline Peritoneal ventriculostomy/External ventriculostomy & 2 \\
\hline Craniosynostosis & 60 \\
\hline Intracerebral genetic therapy & 2 \\
\hline Vertebral laminectomy/Arthrodesis & 5 \\
\hline Craniotomy & 4 \\
\hline Central venous catheter placement & 1 \\
\hline Fixed/attached spinal cord & 1 \\
\hline Moya Moya & 1 \\
\hline Intracerebral tumor resection & 32 \\
\hline Lefort III & 1 \\
\hline Epileptogenic lesion resection & 3 \\
\hline Extradural hematoma drainage & 3 \\
\hline Spinal cord tumorresection & 2 \\
\hline Brainstem tumor resection & 2 \\
\hline Posterior fossa decompression & 1 \\
\hline Intracerebral biospsy & 2 \\
\hline Chiari malformation & 5 \\
\hline Orbital tumor & 2 \\
\hline Frontal cavernoma & 3 \\
\hline Basal skull schwannoma & 3 \\
\hline Cranioplasty & 1 \\
\hline Intraventricular stenting & 1 \\
\hline Scoliosis & 37 \\
\hline Limb amputation & 1 \\
\hline Pelvic osteotomy & 1 \\
\hline Femoral osteotomy & 4 \\
\hline Tumor resection & 2 \\
\hline Femoral prothesis & 1 \\
\hline Vertebral laminectomy/arthrodesis & 1 \\
\hline Interscapular thoracic desarticulation & 1 \\
\hline Total number of patients & 252 \\
\hline
\end{tabular}


Table 2: General Characteristics.

\begin{tabular}{|l|l|}
\hline Characteristic & \\
\hline Median age in months & $62[12.50-144.00]$ \\
\hline Median preoperative hemoglobin levels in g/dL & $11.65[10.38-12.90]$ \\
\hline Median intra-operative hemoglobin levels in g/dL & $9[8-11.03]$ \\
\hline Median postoperative hemoglobin levels in g/dL & $11.2[9.9-12.5]$ \\
\hline Median LOSICU in days & $4[3-10]$ \\
\hline Median LOS in days & $4[2-14.25]$ \\
\hline Median TLOS in days & $9[5-26]$ \\
\hline Median LMV in days & $0[0-2]$ \\
\hline Number of ASA I patients n (\%) & $39(15.48 \%)$ \\
\hline Number of ASA II patients n (\%) & $50(19.84 \%)$ \\
\hline Number of ASA III patients n (\%) & $122(48.41 \%)$ \\
\hline Number of ASA IV patients n (\%) & $36(14.29 \%)$ \\
\hline Number of ASA V patients n (\%) & $5(1.98 \%)$ \\
\hline Emergency n (\%) & $60(23.81 \%)$ \\
\hline Elective surgery n (\%) & $192(76.19 \%)$ \\
\hline Number of patients with complications n (\%) & $83(32.94 \%)$ \\
\hline Re-surgery n (\%) & $21(8.33 \%)$ \\
\hline Deaths n (\%) & $7(2.78 \%)$ \\
\hline Number of transfused patients n (\%) & $195(77.38 \%)$ \\
\hline Number of non-transfused patients n (\%) & $57(22.62 \%)$ \\
\hline Number of abdominal surgical patients n (\%) & $67(26.59 \%)$ \\
\hline Number of neurosurgical patients n (\%) & $137(54.37 \%)$ \\
\hline Number of orthopedic surgical patients n (\%) & $48(19.05 \%)$ \\
\hline Total number of patients N (\%) & $252(100 \%)$ \\
\hline
\end{tabular}

$\mathrm{n}=$ number of patients with the variable; $\mathrm{N}=$ total number of patients.

Table 3: General characteristics in transfusion and no transfusion groups.

\begin{tabular}{|l|l|l|l|}
\hline Characteristic & Transfused patients & Non-Transfused patients & p-value \\
\hline Median age in months & $51[11-130.5]$ & $127[51-168]$ & 0.002 \\
\hline Median preoperative hemoglobin levels in g/dL & $11[10-12.5]$ & $13.2[12.1-14.1]$ & $<0.0001$ \\
\hline Median intra-operative hemoglobin levels in g/dL & $8.5[7.7-10.6]$ & $10.9[9.8-12.0]$ & $<0.0001$ \\
\hline Median postoperative hemoglobin levels in g/dL & $11.2[9.85-12.6]$ & $11.3[10.2-12.1]$ & 0.89 \\
\hline Median LOSICU in days & $5[3-11]$ & $3[1-4]$ & $<0.0001$ \\
\hline Median LOS in days & $8[2-18]$ & $2[1-4]$ & $<0.0001$ \\
\hline Median TLOS in days & $15[5-29]$ & $5[4-7]$ & $<0.0001$ \\
\hline Median LMV in days & $0[0-3]$ & $0[0-0]$ & 0.0001 \\
\hline Number of ASA I patients n (\%) & $32(16.41 \%)$ & $7(12.28 \%)$ & 0.54 \\
\hline Number of ASA II patients n (\%) & $38(19.49 \%)$ & $12(21.05 \%)$ & 0.85 \\
\hline Number of ASA III patients n (\%) & $87(44.62 \%)$ & $35(61.40 \%)$ & 0.034 \\
\hline Number of ASA IV patients n (\%) & $34(17.44 \%)$ & $2(3.51 \%)$ & 0.008 \\
\hline Number of ASA V patients n (\%) & $4(2.05 \%)$ & $1(1.75 \%)$ & 1.0 \\
\hline Emergency n (\%) & $54(27.69 \%)$ & $6(10.53 \%)$ & 0.007 \\
\hline Elective surgery n (\%) & $141(72.31 \%)$ & $51(89.47 \%)$ & 0.007 \\
\hline Number of patients with complications n (\%) & $76(38.97 \%)$ & $7(3.76 \%)$ & 0.0001 \\
\hline Re-surgery n (\%) & $21(10.77 \%)$ & $0(0 \%)$ & 0.005 \\
\hline Deaths n (\%) & $6(3.08 \%)$ & $1(1.75 \%)$ & 1.0 \\
\hline Number of patients n (n/N \%) & $195(77.38 \%)$ & $57(22.62 \%)$ & $<0.0001$ \\
\hline Number of abdominal surgical patients n (\%) & $64(32.82 \%)$ & $3(5.26 \%)$ & $<0.0001$ \\
\hline Number of neurosurgical patients n (\%) & $85(43.59 \%)$ & $52(91.23 \%)$ & $<0.0001$ \\
\hline Number of orthopedic surgical patients n (\%) & $46(23.59 \%)$ & $2(3.51 \%)$ & 0.0002 \\
\hline
\end{tabular}

$\mathrm{n}=$ number of patients with the variable; $\mathrm{N}=$ total number of patients. 
Table 4: Complications.

\begin{tabular}{|c|c|c|c|}
\hline \multicolumn{4}{|l|}{ Complications } \\
\hline Intraoperative complications & $\begin{array}{l}\text { Number of complications in } \\
\text { transfused patients }\end{array}$ & $\begin{array}{l}\text { Number of complications in } \\
\text { non-transfused patients }\end{array}$ & $\begin{array}{l}\text { Number of complications in } \\
252 \text { transfused and non-trans- } \\
\text { fused patients (\%) }\end{array}$ \\
\hline Hemorrhagic shock & 15 & 1 & $16(6.35 \%)$ \\
\hline Bronchospasm /Laryngospasm & 2 & 1 & $3(1.19 \%)$ \\
\hline Difficult intubation & 1 & 0 & $1(0.39 \%)$ \\
\hline Cardiac arrest & 1 & 0 & $1(0.39 \%)$ \\
\hline Anaphylaxis & 1 & 0 & $1(0.39 \%)$ \\
\hline \multicolumn{4}{|l|}{ Postoperative Organ Failure } \\
\hline Neurologic & 16 & 2 & $18(7.14 \%)$ \\
\hline Cardio-circulatory & 13 & 0 & $13(5.16 \%)$ \\
\hline Respiratory & 11 & 1 & $12(4.76 \%)$ \\
\hline Renal & 3 & 1 & $4(1.59 \%)$ \\
\hline Hepatic & 2 & 0 & $2(0.79 \%)$ \\
\hline Multi-organ & 3 & 1 & $4(1.59 \%)$ \\
\hline Hemorragic shock & 1 & 0 & $1(0.39 \%)$ \\
\hline Anaphylaxis & 1 & 0 & $1(0.39 \%)$ \\
\hline \multicolumn{4}{|l|}{ Postoperative infection or sepsis } \\
\hline Pulmonary & 6 & 2 & $8(3.17 \%)$ \\
\hline Abdominal & 7 & 0 & $7(2.78 \%)$ \\
\hline Urinary & 6 & 0 & $6(2.38 \%$ \\
\hline Septicemia & 10 & 0 & $10(3.97 \%)$ \\
\hline neuro-meningeal & 2 & 0 & $2(0.79 \%)$ \\
\hline surgical wound infection or sepsis & 2 & 0 & $2(0.79 \%)$ \\
\hline Total number of complications & 103 & 9 & 112 \\
\hline
\end{tabular}

p-value $<0.0001$.

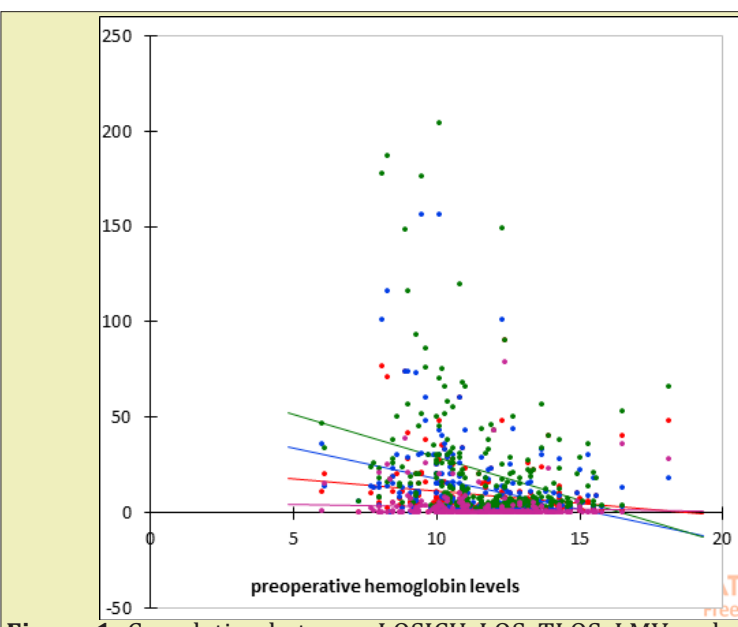

Figure 1: Correlation between LOSICU, LOS, TLOS, LMV and preoperative hemoglobin levels.

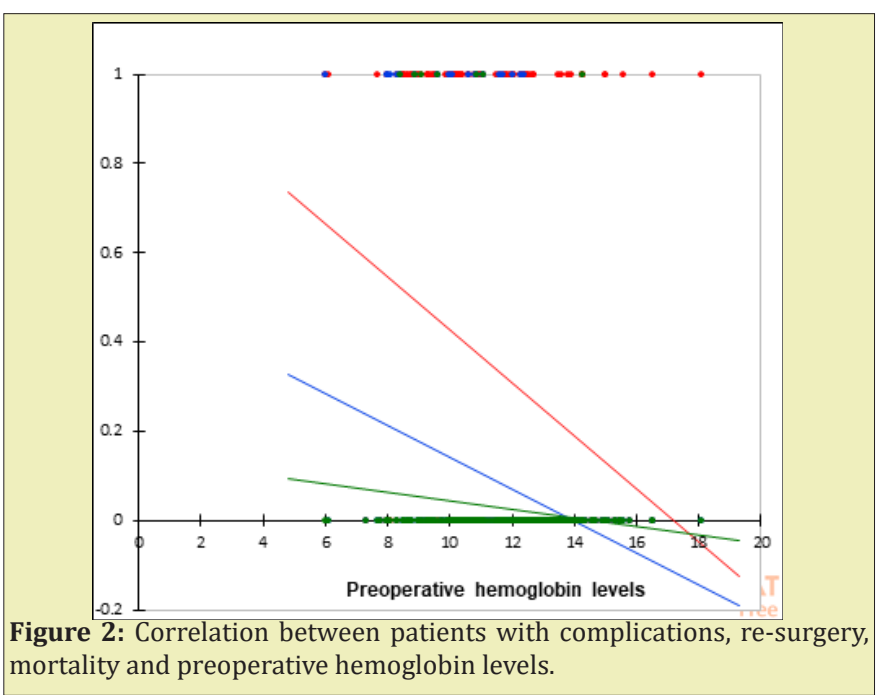


In linear regression, intra-operative hemoglobin levels were negatively correlated to LOS (with $9 \%$ variability, $\mathrm{p}<0.0001$ ) and TLOS (with 5\% variability, $\mathrm{p}=0.001$ ). Low intra-operative hemoglobin levels below $5 \mathrm{~g} / \mathrm{dL}$ were correlated to higher LOS and TLOS. There was no correlation between intra-operative hemoglobin levels with LOSICU ( $p=0.52)$ and LMV ( $p=0.078)$ (Figure 3). There was a negative correlation between the number of patients with complications and intra-operative hemoglobin levels (with 3\% variability, $\mathrm{p}=0.004$ ) (Figure 4). There was no correlation between intra-operative hemoglobin levels and re-surgery $(p=0.23)$ and mortality $(p=0.3)$. In linear regression, postoperative hemoglobin levels were positively correlated to LMV (with $4 \%$ variability, $\mathrm{p}=0.002$ ). There was no correlation between LOSICU ( $p>0.06)$, LOS $(p=0.94)$, TLOS $(p=0.5)$ and postoperative hemoglobin levels (Figure 5). There was no correlation between the number of patients with complications, re-surgery, mortality and postoperative hemoglobin levels ( $p>0.7$ ) (Figure 6).

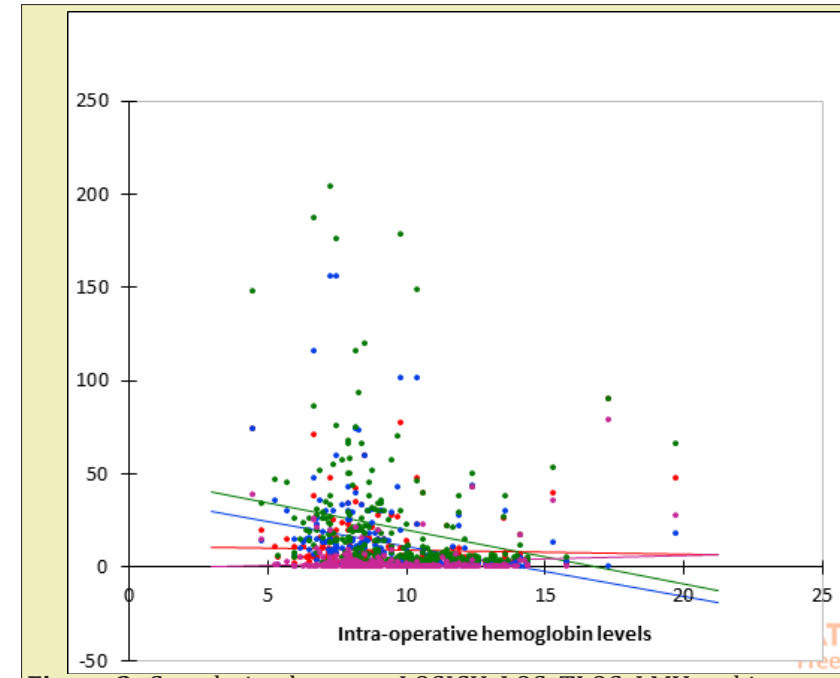

Figure 3: Correlation between LOSICU, LOS, TLOS, LMV and intra-operative hemoglobin levels.

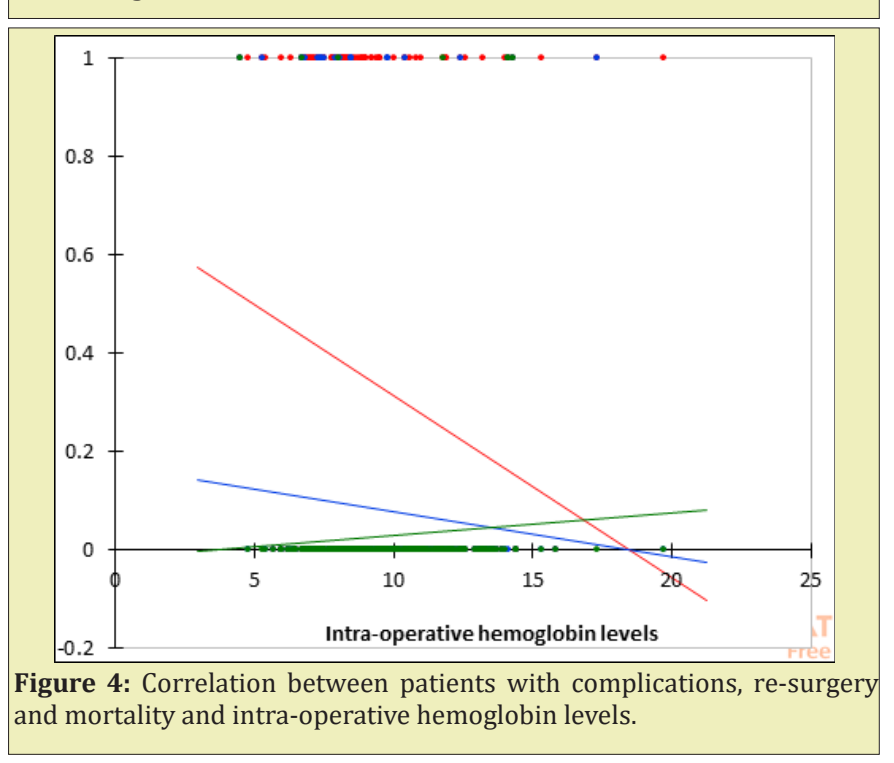

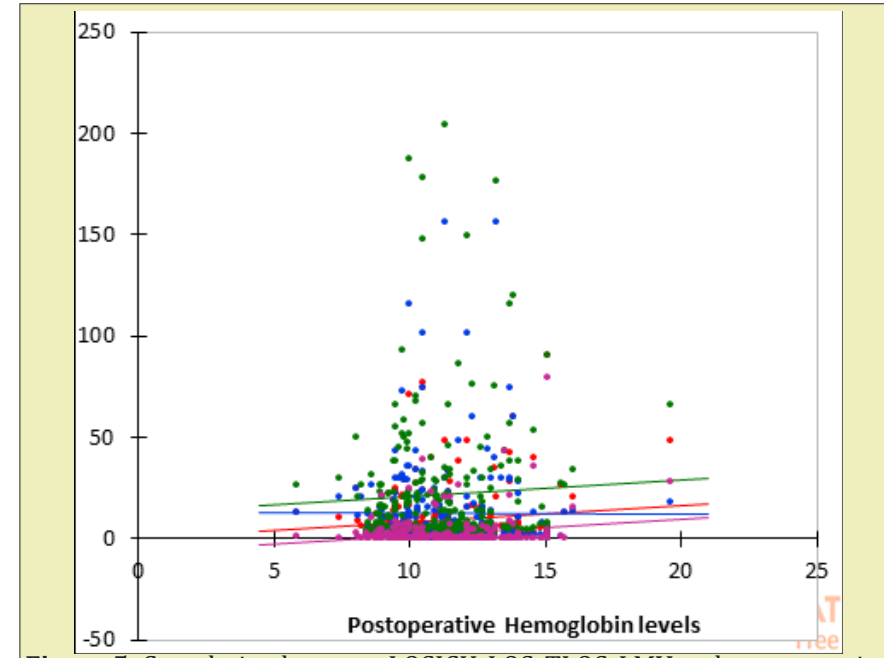

Figure 5: Correlation between LOSICU, LOS, TLOS, LMV and postoperative hemoglobin levels.

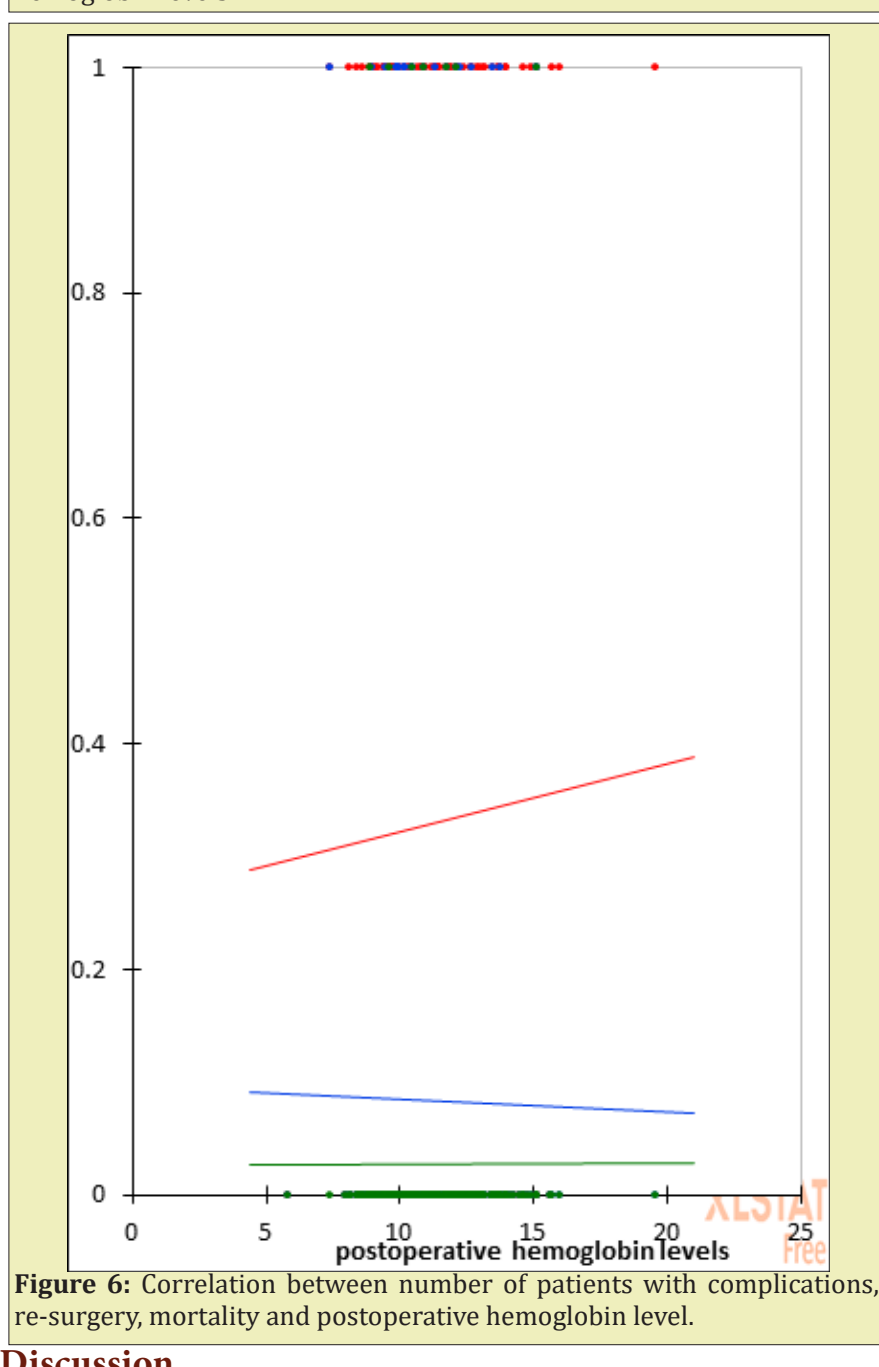

\section{Discussion}

Preoperative hemoglobin levels were significantly correlated to LOSICU, LOS, TLOS and the number of patients with intra-operative and postoperative complications however considering the variability of these outcomes which was between 4 and 9\%, other 
variables also predicted these outcomes as it has been evidenced earlier in the initial studies. ${ }^{1-4}$ In these studies, it was demonstrated that complications, re-surgery, LOSICU, LOS, TLOS, LMV were predicted by the ASA status, transfusion, emergency, age and surgery. Nevertheless, one should not minimize the impact of preoperative hemoglobin levels on postoperative outcome in children. A retrospective study revealed that preoperative anemia was correlated to in-hospital mortality in pediatric non cardiac surgical patients. ${ }^{7}$ It has also been shown that in the general pediatric population anemia was related to mortality and neurodevelopmental disorders. ${ }^{8}$ In this secondary analysis, there was no correlation between preoperative hemoglobin levels and mortality which is not a surprise since in the initial studies, the independent predictor of mortality in this cohort was the ASA score. ${ }^{1-4}$ According to this analysis, preoperative hemoglobin levels less than $6 \mathrm{~g} / \mathrm{dL}$ were correlated to high LOSICU, LOS and TLOS. The transfusion trigger is not absolute and depends on patient's physical status and the clinical context. Transfusion triggers may differ from pre-term patients to older children and also from one clinical situation to another. As it was shown in a study in critically ill children, that restrictive transfusion strategies did not increase the incidence of adverse outcome compared to liberal strategies. ${ }^{9}$ Intra-operative hemoglobin levels were correlated to LOS, TLOS and complications with a variability of 3-9\% which means that other predictors of these outcomes explain the major remaining variability and these predictive factors have been determined in previous studies as stated here above. ${ }^{1-4}$ Extreme values of intra-operative hemoglobin levels of less than $5 \mathrm{~g} / \mathrm{dL}$ were correlated to high LOS and TLOS. Preoperative and intra-operative hemoglobin levels impact thus on recovery after surgery. Postoperative hemoglobin levels were positively correlated to LMV with a variability of $3 \%$ which implies that other factors predicted the majority of the variability of LMV. ${ }^{1-4}$ Probably the positive correlation between postoperative hemoglobin levels with LMV was due to the side effects of transfusion rather than to a high hemoglobin level. ${ }^{5}$

The results of this secondary analysis confirm that postoperative outcome is multifactorial and that preoperative, intra-operative and postoperative hemoglobin levels contributed significantly but there were other predictors of postoperative outcome which played a major role as evidenced in the initial trial. ${ }^{1}$ How do low hemoglobin levels affect outcome in terms of postoperative complications, of LOSICU, LOS, TLOS? As illustrated here above, the most common reported postoperative complications in this cohort were neurologic, respiratory, cardio-circulatory failures and infectious mainly sepsis and septicemia. One possible explanation is that immunomodulation in critical ill patients who are immune compromised predispose patients to organ dysfunction and infection. ${ }^{5}$ As explained here above, the majority of the patients were severely ill as assessed by the ASA score 3 or more. One other possible explanation is that hemoglobin is one of the determinants of oxygen de- livery. If anemia occurs to a point that oxygen delivery is impacted, reduction in oxygen delivery can occur and if oxygen consumption increases more than oxygen delivery, tissular hypoperfusion can occur and therefore organ dysfunction and infection can develop. ${ }^{10,11}$ Organ dysfunction predisposes the patient to ICU admission and to higher LOS. How do postoperative hemoglobin levels impact on LMV? As explained here above, postoperative hemoglobin levels were positively correlated to LMV with a variability of $3 \%$. One possible explanation of this observation is that transfusion may have contributed to LMV as it has been demonstrated earlier that all types of blood products are related to high LMV. ${ }^{1-4}$ LMV in patients with higher hemoglobin levels were correlated to transfusion, which implies that it is not necessary to transfuse to achieve higher hemoglobin levels $(>12 \mathrm{~g} / \mathrm{dL})$ in these children and that probably transfusion indirectly explained the positive correlation between postoperative hemoglobin levels and LMV. Transfusion related lung injury (TRALI) has been reported in transfused critically ill patients. ${ }^{5}$ The results of this study concerned pediatric patients with a median age of 62 months [12.50-144.00] in neurosurgery, abdominal and orthopedic surgery. Preoperative, intra-operative, postoperative hemoglobin levels, patient global status, clinical conditions and the balance between benefits and risks of transfusion and anemia need to be taken into account together when making a decision to transfuse or not to transfuse a pediatric patient. The transfusion trigger or the hemoglobin level alone are not enough to decide whether to transfuse or not. Keeping in mind that low hemoglobin levels below $5-6 \mathrm{~g} / \mathrm{dL}$ are correlated to adverse postoperative outcome in terms organ dysfunction, LOSICU, LOS, TLOS and that transfusion to achieve high hemoglobin levels above 12g/ $\mathrm{dL}$ are correlated to LMV. This emphasizes the need to reconsider transfusion guided protocols to optimize patient blood management and improve outcome in children.

\section{Conclusion}

This study has revealed that preoperative, intra-operative and postoperative hemoglobin levels were correlated to postoperative outcome in terms of organ dysfunction, LOSICU, LOS, TLOS and LMV in neurosurgery, abdominal and orthopedic pediatric surgery. The physiopathology underlying these adverse outcomes are probably explained by:

a) Reduction in tissular oxygen delivery due to anemia which can result in organ dysfunction which can predispose a patient to high LOSICU, LOS and TLOS.

b) Transfusion to achieve high hemoglobin levels and transfusion related immunomodulation in compromised immune patients may predispose patients to organ dysfunction such as TRALI and TACO which can predispose the patient to LMV. Hemoglobin levels are among other multifactorial predictors of postoperative outcome in pediatric surgical patients em- 
phasizing the importance of a global patient blood management implementation program to improve outcome in children.

\section{Acknowledgments}

None.

\section{Funding}

None.

\section{Conflicts of interest}

Author declares that there is no conflict of interest.

\section{References}

1. Kumba C, Cresci F, Picard C, et al. Transfusion and Morbi-Mortality Factors: An Observational Descriptive Retrospective Pediatric Cohort Study. J Anesth Crit Care Open Access. 2017;8(4):00315.

2. Kumba C, Taright H, Terzi E, et al. Blood Product Transfusion and Postoperative Outcome in Pediatric Neurosurgical Patients. EC Anaesthesia. 2018;4(8):288-298.

3. Kumba C, Lenoire A, Cairet $P$, et al. Is Transfusion an Independent Risk
Factor of Postoperative Outcome in Pediatric Orthopedic Surgical Patients? A Retrospective Study. J Emerg Med Critical Care. 2018;4(2):7.

4. Kumba C, Querciagrossa S, Blanc Thomas, et al. Transfusion and Postoperative Outcome in Pediatric Abdominal Surgery. J Clin Res Anesthesiol. 2018;1(1):1-8.

5. Muszynski JA, Philip C Spinella, Jill M Cholette, et al. Transfusion-related immunomodulation: Review of the literature and implications for pediatric critical illness. Transfusion. 2017;57(1):195-206.

6. Kumba C, Querciagrossa S, Harte C, et al. A Systematic Review and Meta-analysis of Goal Directed Intra-Operative Transfusion Protocols Guided by Viscoelastic Methods and Perioperative Outcomes in Children. Int J Recent Sci Res. 2019;10(03):31466-31471.

7. Faraoni D. Relationship Between Preoperative Anemia and In-Hospital Mortality in Children Undergoing Noncardiac Surgery. Anesth Analg. 2016;123:1582-1587.

8. Kumba C. Iron deficiency with or without anemia and perspectives of perioperative management in children. Adv Pediatr Res. 2019;6:33.

9. Lacroix J. Transfusion Strategies for Patients in Pediatric Intensive Care Units. N Engl J Med. 2007;356:1609-1619.

10. Kumba C. Physiology Principles Underlying Goal Directed Therapies in Children. Res Pediatr Neonatol. 4(4).RPN.000591.2020.

11. Kumba C. Rationale of Goal Directed Therapies in Children. Adv Pediatr Res. 2020;7:42. 